\title{
TOWARDS A VIRTUAL HUB APPROACH FOR LANDSCAPE ASSESSMENT AND MULTIMEDIA ECOMUSEUM USING MULTITEMPORAL-MAPS
}

\author{
R. Brumana*a , M. Santana Quintero ${ }^{\text {b }}$, L. Barazzetti ${ }^{a}$, M. Previtali ${ }^{a}$, F. Banfi a ${ }^{a}$ D. Oreni ${ }^{\text {a }}$, D. Roels ${ }^{\text {c }}$, F. Roncoroni $^{\text {a }}$ \\ ${ }^{\text {a }}$ Politecnico di Milano, Department of Architecture, Built Environment and Construction Engineering, Via Ponzio 31, 20133 \\ Milano, Italy - (raffaella.brumana, luigi.barazzetti, mattia.previtali, fabrizio.banfi, daniela.oreni, fabio.roncoroni)@ polimi.it \\ b Carleton University, Civil and Environmental Engineering - mariosantanaquintero@gmail.com \\ ${ }^{\mathrm{c}}$ RLICC (Kul) Master After Master Student, Internship at POLIMI - \\ daphne_roels@hotmail.com
}

KEY WORDS: Landscape assessment, interactive multimedia systems, panoramic images, open data, brokering approach

\begin{abstract}
:
Landscapes are dynamic entities, stretching and transforming across space and time, and need to be safeguarded as living places for the future, with interaction of human, social and economic dimensions. To have a comprehensive landscape evaluation several open data are needed, each one characterized by its own protocol, service interface, limiting or impeding this way interoperability and their integration. Indeed, nowadays the development of websites targeted to landscape assessment and touristic purposes requires many resources in terms of time, cost and IT skills to be implemented at different scales. For this reason these applications are limited to few cases mainly focusing on worldwide known touristic sites. The capability to spread the development of web-based multimedia virtual museum based on geospatial data relies for the future being on the possibility to discover the needed geo-spatial data through a single point of access in an homogenous way. In this paper the proposed innovative approach may facilitate the access to open data in a homogeneous way by means of specific components (the brokers) performing interoperability actions required to interconnect heterogeneous data sources. In the specific case study here analysed it has been implemented an interface to migrate a geo-swat chart based on local and regional geographic information into an user friendly Google Earth ${ }^{\odot}$-based infrastructure, integrating ancient cadastres and modern cartography, accessible by professionals and tourists via web and also via portable devices like tables and smartphones. The general aim of this work on the case study on the Lake of Como (Tremezzina municipality), is to boost the integration of assessment methodologies with digital geo-based technologies of map correlation for the multimedia ecomuseum system accessible via web. The developed WebGIS system integrates multi-scale and multi-temporal maps with different information (cultural, historical, landscape levels) represented by thematic icons allowing to transfer the richness of the landscape value to both tourists and professionals.
\end{abstract}

\section{INTRODUCTION}

Nowadays the Lake of Como, and the Tremezzina, is an attractive mass tourist area, limited to the lake shore and concentrated on very few points, i.e. the Villa Carlotta Museum (http://www.villacarlotta.it) in Tremezzo has 260.000 tourists per year, or famous properties of star and actors (i.e. the Villa Oleandra by G. Clooney). Tourist remain nearby the lake for summer sports. The lack of information and awareness of the richness of the values and traces of the studied area leads to a deserted environment in fall and winter. According to the European Landscape Convention (2000) to raise the awareness of every landscape, to propose specific measures for the identification and assessment (Sites, 2008), and to fulfil the JRA objectives, stating the important link between the role of connecting people for the growing of a reflective society and knowledge creating in safeguarding cultural heritage; the objective of this research is to identify an assessment methodology, correlating and managing, as many of the remained traces, the viewable, less viewable and the untraceable values experimenting a sort of Geo-SWOT chart, combining such values with metric and non-metric multi-temporal-multiple view maps, ancient and current (Figure 1).

To reach this goal an interactive navigation system has been developed within the "Multimedia system of the landscape of the Municipality of Tremezzina on the Lake of Como', opening on October 2015 in the Villa Mainona. The general aim of this work carried out with the contribution of international universities, is to boost the integration of assessment methodologies with digital geo-based technologies of map correlation for the multimedia eco-museum system accessible via web.
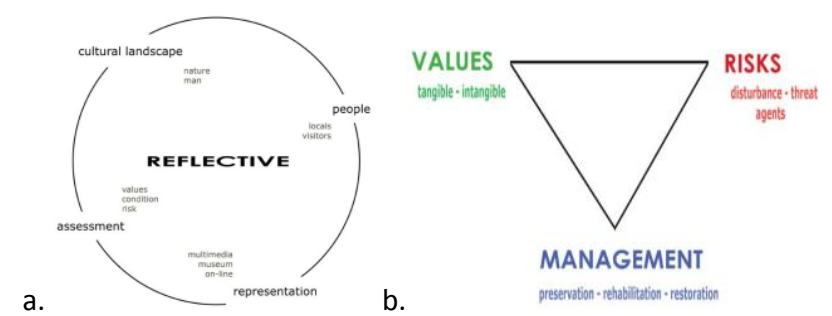

b. MANAGEMENT
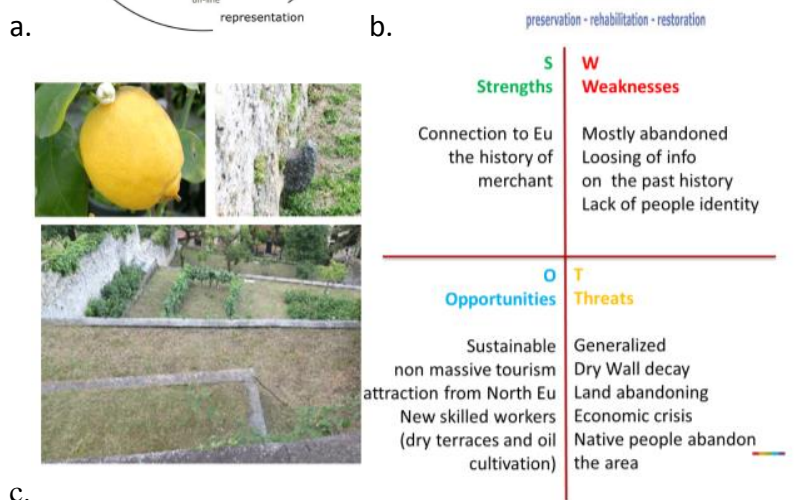

c.

Figure 1. The Reflective approach for cultural heritage preservation and valorisation. 
In particular, the developed system has been designed, taking into account the built environment as a system: sum of natural transformation, ancient artefacts stratification, and human activities partially covering the tangible traces as a vehicle of intangible values comprehension. Such approach could contribute to enhance the landscape analysis and preservation in the future intervention, and increase the participation processes by tourist and local people within a "reflective society" as stated by the European SRA (Strategic Research Agenda). The developed system integrates multi-scale and multi-temporal maps with different information (cultural, historical, landscape levels) represented by thematic icons (historical hamlets, ancient paths, vaulted passages) allowing to transfer the richness of the landscape value to both tourists and professionals (Figure 2).

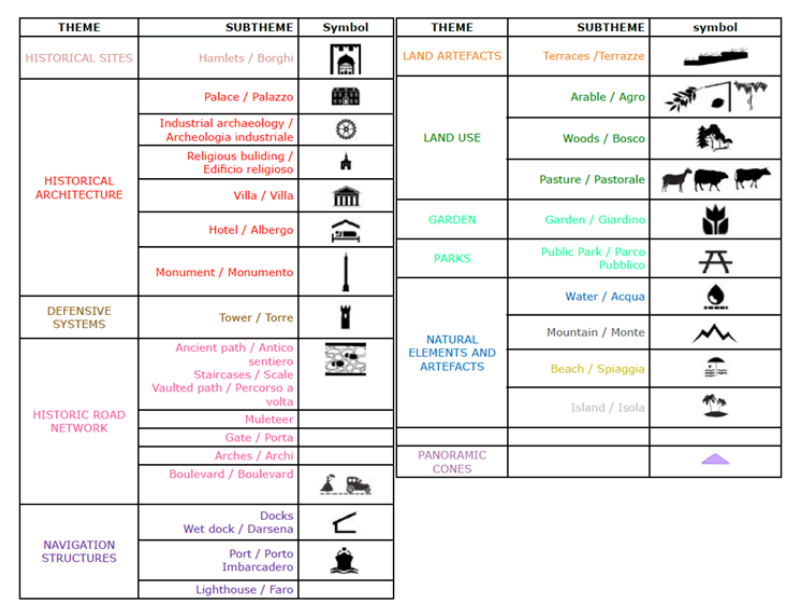

Figure 2. The thematic icons used to represent the themes and sub-themes of the Tremezzina.

\section{THE ASSESSMENT METHODOLOGY}

The PA (Province of Como and Municipality of Tremezzina) are promoting researches on the past periods to transfer the knowledge of the rich history of this territory to the people. In this contest the Ecomuseum project aims to valorise the rich history of the Tremezzina region and transfer such awareness to people, both local inhabitants and tourists, by using innovative tools and geobased applications.

The "Tremezzina" has a rich stratified history which had a significant impact in the evolution of the landscape that the Ecomuseum aims to valorise by spreading knowledge among the people visiting the area.

In particular, four main historical phases can be highlighted in the timeline to be valorised by the multimedia initiatives (Figure 3a):

- the Roman Period: one of the most important roman roads from Aquileia to North Europe and to the Rhine region passed along to the east part of Lake Como (Strada Regina and Via Regia, nowadays mostly destroyed, were two Roman ways crossing Alps passing in the Tremezzina Area),

the early middle age: its strategic position is important for the connections between the two sides of the Alpine region, with development of settlements and defensive systems and religious sites (i.e. the Comacina Island has been inserted in a tourist project with the realization of an Antiquarium in front of the isle);

- $\quad$ the end of the 16th till to 18th century: a new mostly unknown period started due to the emergence of merchants to overcome the economic crisis and the plague during the XVI century, beginning to sell lemons across Europe. They became rich merchants and they were present in the most important markets: thanks to the import of spices and cotton by the East India Company their economic activities increased, and the network became denser. They started trading spices, tobacco and goods. Several merchant families bought warehouses in the Amsterdam harbour to stock their goods and to distribute it building a dense network in Frankfurt, Strasbourg, etc. Some merchants became bankers and borrowed money to royal families. The rich merchants started building palaces in their original countries and across Europe. These palaces are a key element characterizing the hamlets of the Tremezzo and its hillside background level. The decadence of this trading economy at the end of the 18th century, caused their rural use, a decrease in agriculture due to the abandoning of cultivated lemon terraces, and a pour use of the palaces;

the XIX-XX centuries: hotels on the lake were built for the tourists and royal families arriving from Europe, due to the intrinsic beauty of the landscape and the mild climate. Connected to this epoch we can find some characterizing Belle Époque architectures, gardens and villas on the lakeside.

Such a variety of scenarios, architectures, uses, and themes, characterizing the Tremezzina landscape have been taken into account to valorise its rich history.

The landscape is characterized by three different layers representing a complex structure of arable-wood-pasture landscape (Scazzosi, 2004), that give to the landscape a deeper dimension, enlarging the mostly known dimension of the lakeside, inclusive of all the human and natural traces. Three different layers related one to the other have been defined as characterizing the landscape (Figure $3 b$ ); these layers, as discussed in the next section, have been used to address the design of the multimedia museum:

Layer I (cyan in Figure 3b) - Water view front layer, characterized by the historical settlements and Villa systems (XIX-XX centuries), along the ancient road partially destroyed by the pressure of the actual road system;

- $\quad$ Layer II (magenta in Figure 3b) - Hills and Hamlets, with the beautiful unknown hamlets, characterized by lack of preservation and lack of awareness by the local people and by the tourist. However, many traces of a dense network of cobbled ancient paths, vaulted passages under the palaces, historical land use traced by its artefacts (i.e. terraces on the hill slopes, paths, water sources, canalization, drywall), and ancient lemon terraces cultivations, now almost abandoned, are still viewable;

Layer III (green in Figure 3b) - Mountains, with the old pathways for the seasonal grazing and pasture transfer (muleteer). These almost unknown pathways could be inserted in a local centred sustainable 
agriculture, increasingly growing in Lombardy Region (i.e. sheep wool and cheese production).

The background and front ground layers and related values, strengths and weak points, coming from assessment landscape approaches and models (i.e. Nara grid, Van Balen, 2008), are here progressively integrated combining different maps through a georeferenced SWOT chart read on the three multi temporal map layers here considered. The SWOT analysis was used as a basis, distinguishing the strengths/opportunities from the weaknesses/threats by dividing the positive from the negative aspects. The assessments can be useful for decision-makers and make it direct clear for the non-experts, e.g. the local people and tourists. To gather this information together, a geo-SWOT chart was created that contains the different landscape features with tangible traces, intangible values, highlighting potentials and weaknesses, because without saving all the material traces of the past, the vehicle for the richness of all the intangible values will get lost. The timeline with the main historical phases is transferred to the people, in a Reflective approach, together with the georeferred SWOT assessment, including the potentials, pressures and risk evaluation of the built environment.

In this system, the current 'panoramic cones', ancient water view fronts, and quasi-perspectives maps, acquired by privileged observer points, are elements to the identity values for the people in the centuries and intangible signs. Can also play a role in the Impact Valuation of the Intervention, and preservation, being the immediate recognition of the places by citizen (Pereira Roders and Van Oers, 2010). Non metric maps, such as the ancient view front maps, has been correlated to current panoramic maps, acquired and processed through on growing easy to use algorithms and tools, thus allowing to navigate the past and the current views; also, combined with metric georeferred historical maps (ancient cadastral maps, XVIII century) and 3D current spatial data, they can contribute in identifying and georeferencing new traces and signs.
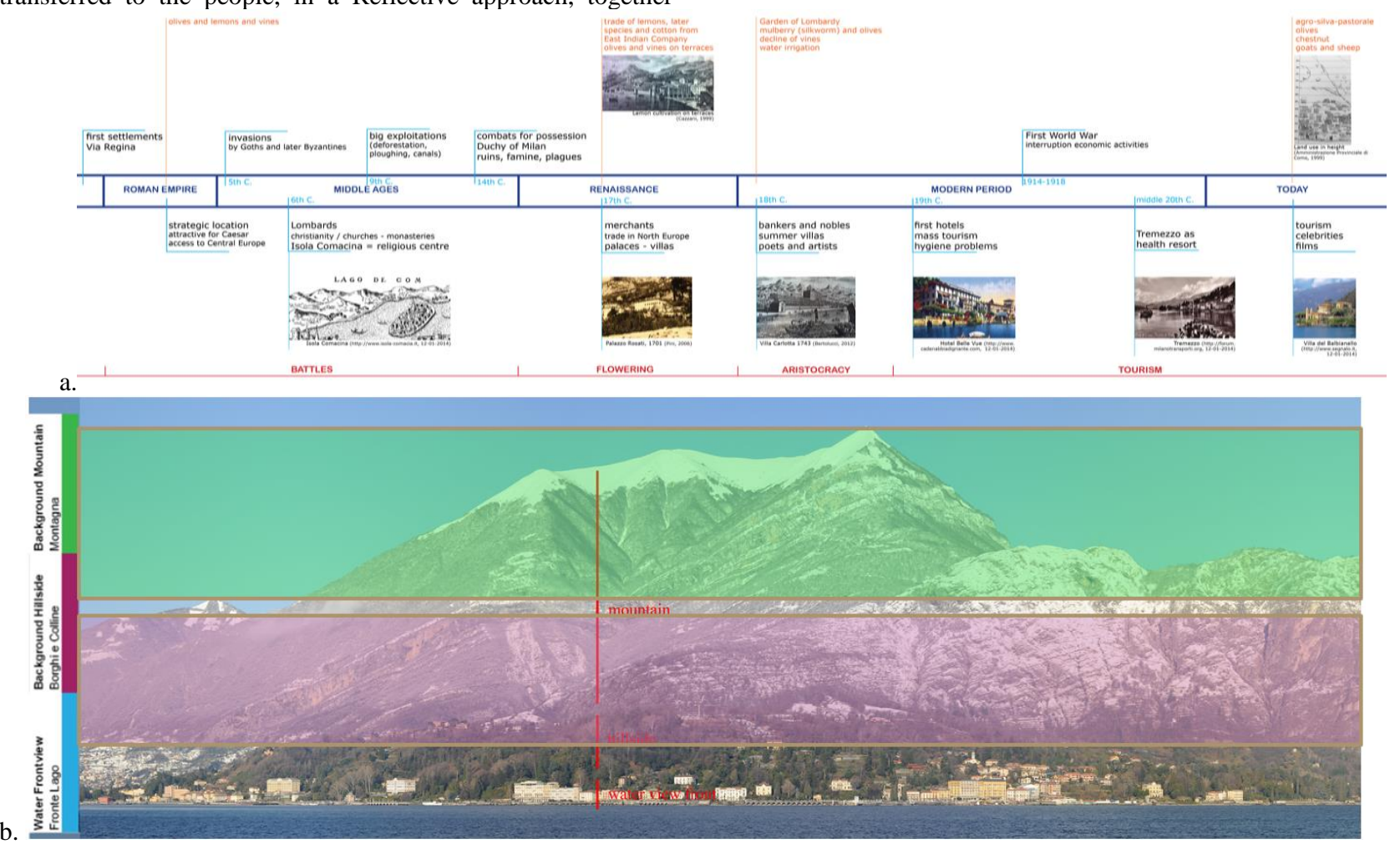

Figure 3. The four main historical phases of the Tremezzina (a) and the arable-wood-pasture landscape three layers framework (b).

\section{DESIGNING A VIRTUAL MUSEUM FOR LANDSCAPE VALORIZATION: THE MULTIMEDIA SYSTEM OF THE "ECOMUSEO DELLA TREMEZZINA"}

The 'New Museology' raised up at the end of the 20th century has great potentialities to help understanding the landscape and valorise historical contents. Indeed, the development of tools for different audience to allow own interpretations widespread in recent museum outfits. In particular, virtual museums can develop personalized, immersive, interactive experiences that enhance our understanding of the world around us. Recently they have become very popular and widespread: online (e.g. Rome Reborn, see also Dylla et al., 2008), in museums (e.g. Provincial Archaeological Museum of Ename, The virtual museum of the ancient Via Flaminia) and on heritage sites. The purposes of these museums are education and research, enjoyment and edutainment, enhancement and experience of the visitors.

The "Ecomuseo della Tremezzina", developed in collaboration with public institutions (Province of Como, Union of municipalities of Tremezzina) and private companies (NEMES, http://www.gesto.biz), is a fixed exhibition devoted to valorise the landscape values through EU ecomuseum. The aim of the museum is to use innovative multimedia systems to create an added experience combining the geographic support within interactive systems.

Different innovative map data sources are here used to help local and tourists in the reading of values and to support the GeoSwot chart: in addition to the traditional bird sight maps and 3D maps, it has been realized a number of "panoramic 
cones', correlated to ancient water front views. Ancient and current quasi-perspectives maps, acquired by privileged observer points, are considered an important vehicle helping people to recognize and identify the themes and subthemes detected and to position the tangible traces and recognize the related intangible socio-economic values of the place. They can play a role in the Impact Valuation of the Intervention, and preservation, being the immediate recognition of the places by citizen (Pereira Roders and Van Oers, 2010).

A Multimedia Museum has been designed as strategy to convey this information and attract people to the landscape preservation theme. As a consequence of the above discussed developments in museology an interactive interface seemed the most convenient to create an enjoyable and educational experience. In particular, a touch screen solution seemed the most adequate for interaction. Indeed, the personal interaction creates more attractiveness and interest, which leads to better memorizing and more appealing results. Nowadays touch technology is quite user-friendly since it is widespread in smartphones, tablet etc. In addition, allows an easy access to information, can display high definition images, videos, and can promote an effective communication. The content can be quickly updated which is a benefit to anticipate with the dynamics of the landscape. This system is connected to a projection screen, that is a passive technique but very handy to visualize information to people in the museum which are not operating on the touch screen.

On the touch screen there are different sections that the visitor can choose:

- $\quad$ Historic maps: a sections visualizing the historic cadastral and water view front maps;

Historic geo-navigation: historic water view front map correlated to the current orthoimage of the lake;

Panoramas - today and in the past: the historic and current water view fronts correlated (Barazzetti, et al., 2013) each other, displaying the 3 landscape layers, with themes and subthemes of the territory;

Tremezzo itineraries on Google Earth $^{\odot}$ : Google Earth $^{\odot}$ interface presenting the themes and subthemes of the territory in a 3D 'Virtual Globe' allowing exploration and navigation; the historical cadastres have been georeferenced (Catasto Teresiano XVIII, Lombardo Veneto XIX) following the open geoportal experiences (Oreni et al, 2012);

Tremezzo and Europe: Google Earth $\odot$ interface presenting the Eu geo-tree of the merchant families;

Ciak on the Lake: a section presenting the location of famous movies along the Como Lake;

Isola Comacina: the historical site of the Isola Comacina, viewed by drone (Brumana et al., 2103).

A special remark has to be pointed on some sections. Old and current panoramas shows the water view front maps: the historic one and the new water front). The potential of the panoramic views integrating multiple views have been underlined in the impact evaluation (The Landscape institute, 2002) and understanding landscape (Brumana et al., 2102) by using low altitude UAV or ground images at a low observer point (Barazzetti et al.,2014). This two views are correlated among them by means of homologous recognized points. It means that while navigation one of them also the second one is navigated of the same quantity. Obviously since the two maps were taken from different viewpoints the velocity of navigation of the two maps changes pointwise. The interactive table contains the same icons for all subthemes in the section. So the visitor can choose the subtheme they want to know more about. For example if the visitor is interested in the villas, the different villas are highlighted on the view fronts on the touchpad along with their characteristics and on which 'landscape layer' they are located. The geo-SWOT chart is the basis for the information on the touch screen.

Due to the fact that not all the precious material traces of the hamlets, the palaces, the ancient paths, and artefacts, can be viewable on the panoramic images, an eye bird 3D GIS view (Esri ArcGIS@) has been generated collecting the historical cadastres, image and videos acquired, and the themes identified across the three layered landscape. GIS can be an important communication tool to help the tourist trips and visiting the hamlets. Thus, in the section Tremezzo itineraries the GIS information was transferred on Google Earth $\odot$ in a 3D virtual globe environment, being already known to the people, reducing the gap a new tool may generate.

\section{ENERGIC OD: THE BROKERING APPROACH}

The geographic contents presented in the museum will also be available on mobile devices (e.g., smartphones and tablets) allowing citizens to access museum contents directly onsite. As previously discussed several Open Data (OD) are required for the valorisation of the landscape. In many cases these data are published by different operators giving rise to a large variety of standards and protocols adopted by different data providers. This is an important barrier for an effective utilization of OD for production of services for citizens. A solution able to reduce the interoperability problems between data providers and applications requires extending the SOA archetype by introducing a new component that interconnects the different service protocols and standards, mediating their models and interface specifications. In addition, this component must work out all the necessary distribution and virtualization capabilities to lower entry barriers. In ENERGIC OD, this intermediary role is played by an infrastructure called Virtual Hub, realizing a Brokering approach (Nativi et al., 2012; Santoro et al, 2012; Nativi et al., 2013).

In a traditional $\mathrm{C}-\mathrm{S}$ architecture, a Server provides services to the Client and each Client sends requests to the Server. In the case the same interface(s), i.e. the same application, the need to access multiple services and the interaction with these services have to be implemented in a separate way. This means that multiple standards and protocols may be implemented by the application developer.

The brokering-oriented architecture changes this architectural style by requiring the use of a specific intermediary: the brokering layer. The broker de-couples the server and the client layers allowing the C-S separation that is crucial for accessing multiple services and Open Data developed by specific user communities. On the other hand, the effort of the application developer to access heterogeneous sources is much reduced. In fact, the brokering architecture moves the implementation of standard and protocol mediation to the brokering layer.

The C-S decoupling simplifies the interoperability requirements for both server and client components, improving scalability and flexibility. Client and server components can evolve independently and interoperability problems are solved inside the mediation layer. In particular, clients can access to the OD in the same way regardless the Server interface changes. The brokering architecture, facilitates architecture evolution of both clients and servers since all protocols and interface changes are solved by the broker. Even if the interface of a Server changes 
significantly, the Client access of data remain unchanged. Giving always the same interface a Virtual Hub approach give the chance to solve many interoperability problems allowing also the reuse of OD, which is one of the major concern of Public Authorities producing and delivering OD.

A broker is a mediator (Figure 4a) which implements some services (i.e. discover, access, distribution, added value, etc.) to facilitate the interconnection between client and server components in a Client-Server architecture. In particular, the broker which will be developed in ENERGIC OD project will present the following functional modules:

Client request distribution in asynchronous way. This implementation is performed to allow a client to distribute requests for searching resources across more data providers. One of the major concerns is the waiting time for a query. Indeed, one of the primary disadvantage of a brokering systems is the userperceived performance reduction (Fielding, 2000). One of the strategies to increase performance is applying shared caching at the brokering to replicate the result of an individual request such that it may be reused by later requests. Discover of OD is enabled by specific services (e.g., search engines) and by appropriate ancillary information (i.e., metadata profile ISO 19115) associated to the resources, independently from their structure.

$\mathrm{C}-\mathrm{S}$ interface protocol adaptation. This includes functionalities to match many server protocols and client ones, and vice-versa. Access services support electronic data retrieval, usually based on spatial constraints and other criteria, in forms useful for client-side processing. Supported backend for data sources access includes: OGC standard services (e.g., WMS, WFS, WCS, SOS, CSW), ESRI ArcGIS Geoportal catalog service, THREDDS, etc. In the frontend the broker can be accessed by using a JavaScript API. This API exposes the brokering functionalities (semantic discovery and dataset access) hiding its complexity. In this way an easy programming for most common operations is allowed. However, full interfaces and options to the broker are available.

Added-value functionalities at the C-S interconnection level: The ENERGIC OD broker will implement advanced/semantic discovery. In particular, a semantic broker will automatically looks for synonyms during a search so that researches are extended. In particular, two different strategies will be used: (i) automatic query expansion, keywords are expanded automatically by the broker by interrogating a set of aligned semantic instruments (typically, controlled vocabularies, thesauri, gazetteers, and ontologies). The final result consists of a set of semantically related queries that are all executed by the discovery broker. In the (ii) user-assisted query expansion, i.e. the second discovery style, we applies the same strategy, but the semantically-related terms, retrieved from the aligned semantic instruments, are presented to users who are allowed to browse a graph developed according to these terms and select the most pertinent terms.

Transformation module will solve for spatial inconsistencies and access data having different CRS and perform data integration in the same CRS.
As discussed, the introduction of the Brokering layer shifts the C-S architecture from a two-tier (Client-Server) to a three-tier (Client-Broker-Server) configuration. In this schema the broker may became a single point of failure. In particular, updating, governance and maintenance of the brokering are a significant problem to be evaluated. However, this is a common problem when complexity is shifted from Users and Resource Providers to the infrastructure/platform.

The system architecture for the developed app is presented in Figure $4 \mathrm{~b}$. The application can be accessed by all browser supporting HTML5 and allows for the visualization of open data relevant for landscape valorisation directly on the mobile.
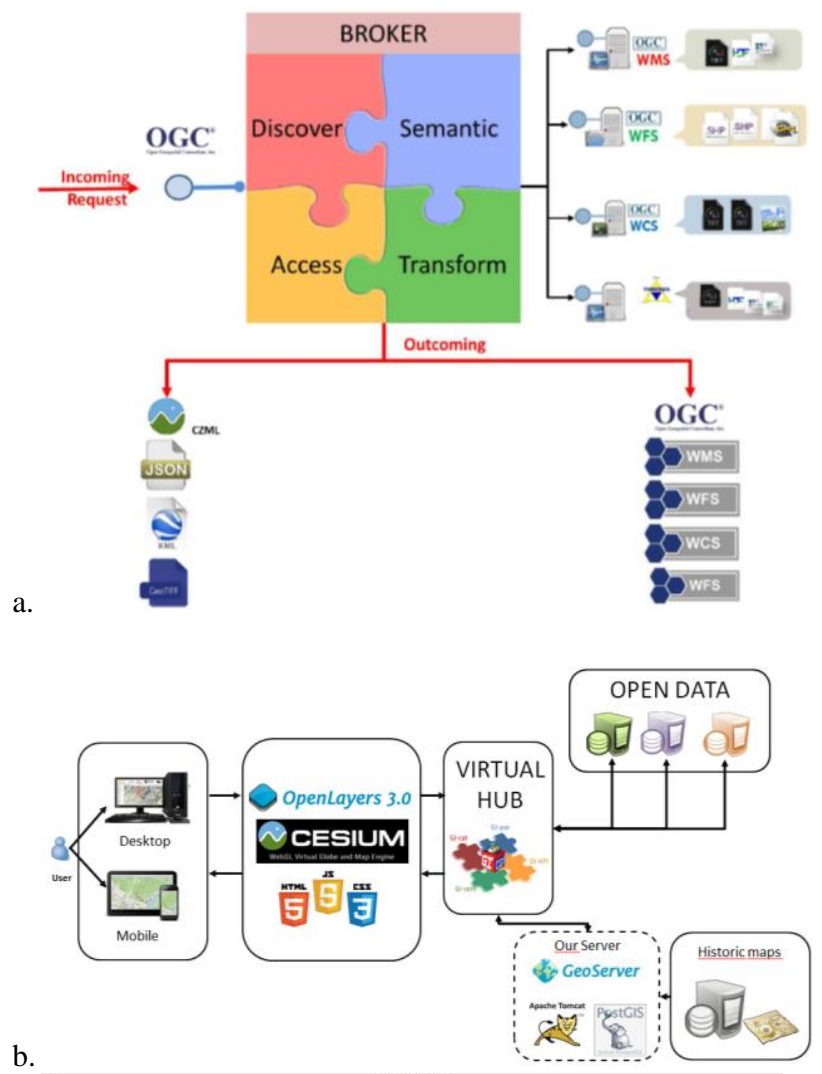

b.

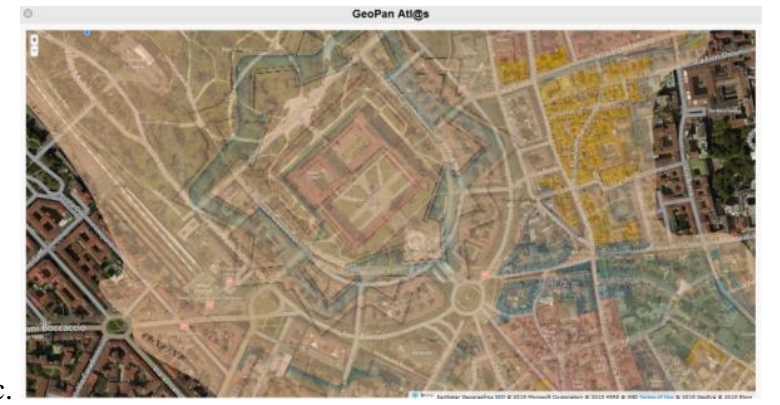

Figure 4. The broker architecture for heterogeneous data discovery and access (a), the architecture of the developed mobile application (d) and the visualization of historical maps trough the developed application (c).

\section{CONNECTING PEOPLE: THE GOOGLE EARTHC 3DGIS VIRTUAL MUSEUM AND THE PROPOSED ITINERARIES SHARED ON SITE BY I-DEVICES}

The purpose of the "Ecomuseo della Tremezzina" is to raise the awareness of heritage and to improve the participation of the people to the landscape valorization. In this sense a well-known 
platform may increase the participation of the public and make the information accessible for as many people as possible, in order to help people in connecting the virtual navigation with the real on site visit, supported by I-device and the tool developed (Santana Quintero and Addison Alonzo, 2007). All themes and subthemes along with pictures organized as different layers in a GIS system were converted into KML files. The possibility to display the themes and the subthemes of the territory along with some suggested itineraries may encourage people to explore the territory and make their choice according to their own interests. Historic cadaster maps are a powerful tool to understand the evolution of a place, for this reason their availability to the Tremezzo itineraries along with the themes of the territory was included. Once georefenced all the cadastral maps (Barazzetti et al., 2014), they were uploaded in the Google Earth $^{\odot}$ (Figure 5).
The ancient cadastral maps are overlaid to actual maps, in this way people can directly discover the territory changes. In addition, the Google Earth 3D view gives an interesting approach to the old maps and can attract more the people attention. A common QRcode (Figure 7) has been implemented and it will allows at the official opening of the museum a link to the application, implemented by using the Virtual Hub approach, accessible by all the visitor not only inside the museum but especially during the on-site trip itineraries suggested in the museum, viewable by their own devices (Smartphones, IPad, tablets). Indeed, many projects use phone applications to improve the research and the experience (Cyark, Mosaic, Historypin, Heritage Passages, etc.).
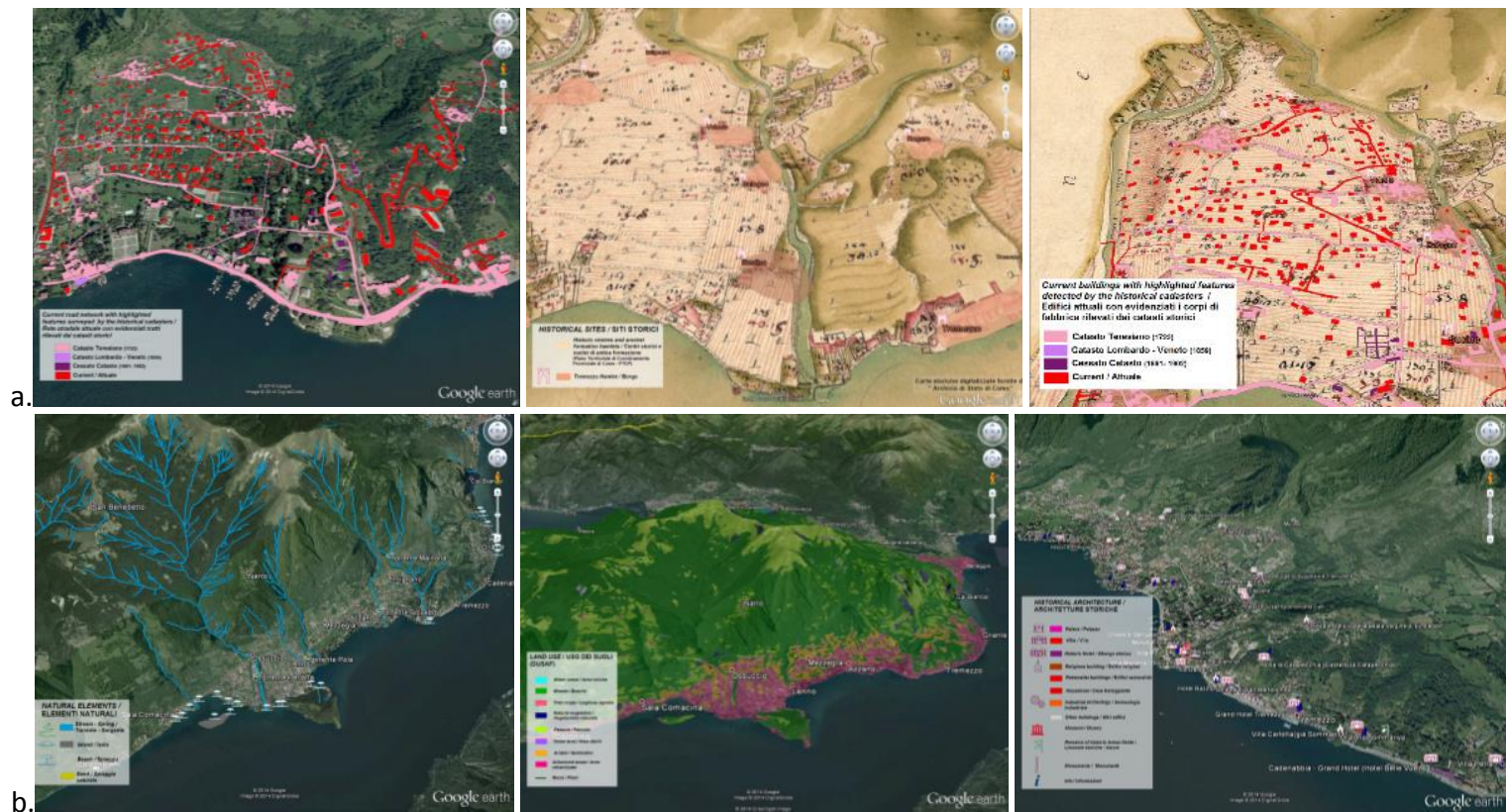

Figure 5. Tremezzo itinerary section with some themes and subthemes of the territory presented: Ancient roads (a), Historic sites and historic cadaster (b), Natural elements (c), Land use (d), Historic Architecture (e), Defensive systems (g), georeferenced images

(h).

A remark need to be pointed out on the Geotree section (Figure 6). It was noticeable, but unknown to the public, that many important families of merchants and aristocrats lived in Tremezzo.

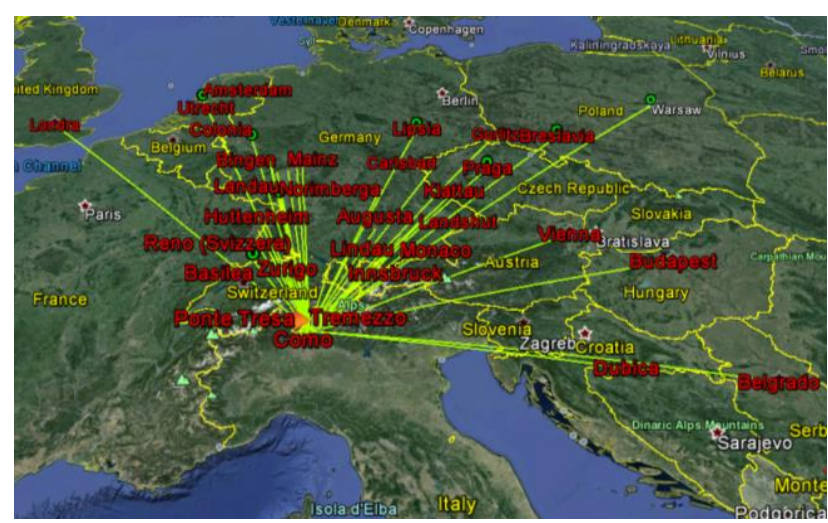

Figure 6. Tremezzo Geotree section.

They had connections all over North-Europe and can be linked to some important cities. Several families are better known in other countries, while they originally come from Tremezzo. To improve the knowledge about these interconnections it is necessary to find a way to represent them. For this reason a geotree was created to visualize all these connections in Google Earth $(\odot)$ environment. Thanks to the richness and patient archive work made during these years by the historic-artistic guidebooks of Tremezzo (Pini, 2003 and Pini, 2006) all the information on the Tremezzo families were localized in the Virtual Globe. All these locations are connected to Tremezzo. By analyzing theme, it is clearer to understand the 'patterns'. In particular, it is quite evident that a large number of merchants had emporiums along the Rhine river. By clicking on the georeferenced points identified further historical information are provided.

\section{CLOSING REMARKS}

The as-found situation of the proposed case study has been digitally virtualized and augmented by the geo-SWOT content, which can have a high potential in the dissemination for a large public contributing to create 'mindscapes', landscapes with a sensitive side reflecting the generated feelings in the 
environment, supported by multimedia tools to visualize and spread information. People - visitors and locals - can appreciate the tangible traces and intangible ones, and the variety of strengths of the three different layers of cultural landscape, connecting the hamlet to the waterfront. The aim is to improve their knowledge in order to safeguard the tangible and intangible values of Tremezzina. A direct participation of the local inhabitants will improve the sustainable maintenance and the survival of tangible and intangible values. Attracting tourists to the different landscape levels by providing virtual and on site itineraries, extending the museum information through a geographic application accessible by smartphones and tablets, can contribute to improve a sustainable informed touristic development of Tremezzina also in the winter and fall.
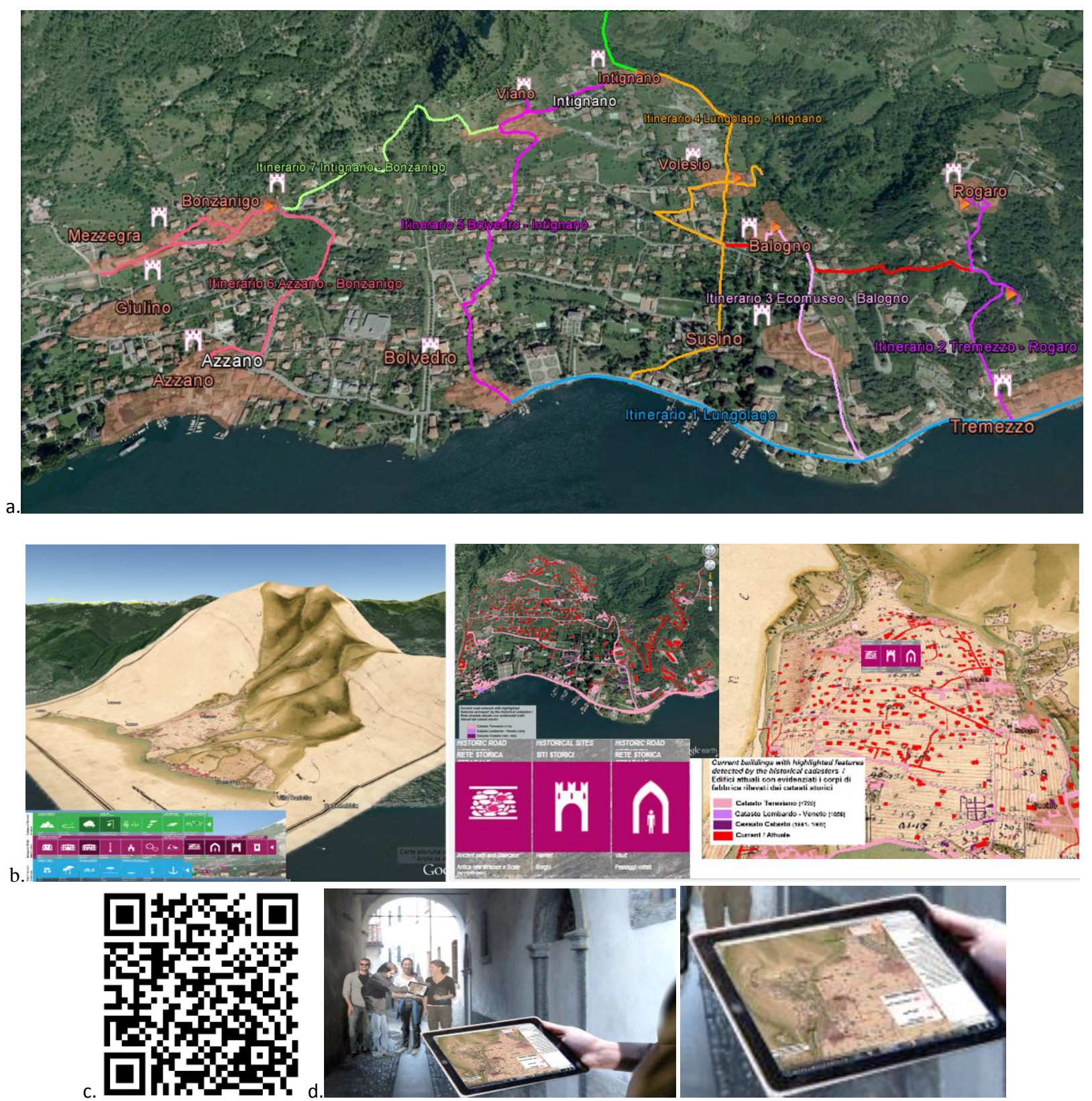

Figure 7. Tremezzo itineraries accessible online (a) along with some other thematic layers (b). The QR code to access application contents (c) and iPad visualization (d)

\section{ACKNOWLEDGEMENTS}

The research leading to these results has received funding from European Commission Competitiveness and Innovation Framework Programme (CIP) under grant agreement $n^{\circ} 620400$ for the project ENERGIC-OD (European NEtwork for Redistributing Geospatial Information to user Communities Open Data).

\section{REFERENCES}

Barazzetti L., Brumana R., Oreni D., Previtali, M., 2014. Historical Map Registration via Independent Model Adjustment with Affine Transformations. In ICCSA 2014 (pp. 44-56). Springer Int. Publishing

Barazzetti L., Brumana R., Oreni D., Roncoroni F., 2013. Recognizing landscapes: can we change the point of view of 
geographic data?) In: JOURNAL OF MOBILE MULTIMEDIA, scopus indexed, Vol. 9(1-2), 2013, pp. 39-52

Brumana R., Oreni D., Van Hecke L., Barazzetti L., Previtali, M., Roncoroni, F., Valente, R., 2013. Combined Geometric and Thermal Analysis from Uav Platforms for Archaeological Heritage Documentation. ISPRS Annals, 1(1), 49-54.

Brumana, R., Scaioni, M., Barazzetti, L., Cuca, B., Oreni, D., and Alba, M., 2012. Panoramic UAV views for landscape heritage analysis integrated with historical maps atlases, Selected Paper CIPA 2011, Geoinformatics FCE CTU 9, Prague, 2012, pp-39-49

Dylla K., Frischer B., Mueller P., Ulmer A., Haegler, S., 2008. Rome reborn 2.0: A case study of virtual city reconstruction using procedural modeling techniques. Computer Graphics World, 16, 25.

Nativi, S., Craglia, M., Pearlman, J., 2012. The brokering approach for multidisciplinary interoperability: a position paper. International Journal of Spatial Data Infrastructures Research, 7, 1-15.

Nativi, S., Mazzetti, P., Geller, G. N., 2013. Environmental model access and interoperability: The GEO Model Web initiative. Environmental Modelling \& Software, 39, 214-228.

Oreni D., Brumana R., Cuca B., 2012. Digital Cartographic Heritage in Service to the Society: Landscape Analysis for Informed Decision Making. In: Eds. Gabriele Guidi, et al. Virtual Systems in the Information Society. p. 499-506, NJ, USA: IEEE, ISBN: 9781467325622, Scopus Ind. 084872049602, Milan (Italy), 2 - 5 September 2012

Pereira Roders A. and Van Oers R. 2010. Outstanding Universal Value, World Heritage Cities Sustainability: Mapping Assessment Processes, In: Offenhaufer Dieter, Zimmerli Walther Ch., Albert Marie-Theres (Eds.), World Heritage And Cultural Diversity: German Commission for UNESCO, 2010, p. 225.

Pini L., 2003. Tremezzo: il paese dove fioriscono $i$ Limoni. Guida storico-artistica, Milano: SilvanaEd.

Pini L., 2006. Tremezzo: il fascino ei borghi. I palazzi, le ville, le filande. Guida storico-artistica, Milano: SilvanaEd.

Santana Quintero M. and Addison Alonzo C., 2007. Digital Tools For Heritage Information Management and Protection: The Need Of Training, In: Wyeld T.G., Kenderdine S., Docherty M. (Eds): Vsmm 2007, Lncs 4820, Springer-Verlag Berlin Heidelberg, 2008, Pp. 35-46.

Santoro, M., Mazzetti, P., Nativi, S., Fugazza, C., Granell, C., \& Díaz, L., 2012. Methodologies for augmented discovery of geospatial resources. Geographic Information Systems: Concepts, Methodologies, Tools, and Applications: Concepts, Methodologies, Tools, and Applications, 305.

Sites, O. C. H., 2008. The ICOMOS Charter for the Interpretation and Presentation of Cultural Heritage Sites. International Journal of Cultural Property, 15, 377-383.

Scazzosi L., 2004. Reading and assessing the landscape as cultural and historical heritage, Landscape Research 29 (4), pp. 335-355.
The Landscape Institute, Institute of Environmental Management \& Assessment, Guidelines for Landscape and Visual Impact Assessment, Spon Press, London, 2002

Van Balen K., 2008. The Nara Grid: An Evaluation Scheme Based on the Nara Document on Authenticity, APT, Bulletin: Journal of Preservation Technology 39 (2-3), 2008, pp. 39-45. 